\title{
Understanding B-Site Disorder in HAADF-STEM Images of Double Perovskite Thin Films Using the Quantum Excitation of Phonons Model
}

\author{
Bryan D. Esser ${ }^{1}$, Adrian J. D’Alfonso ${ }^{2}$, Manisha Dixit ${ }^{1}$, Robert E. A. Williams ${ }^{1}$, Hamish L. Fraser ${ }^{1}$, \\ Leslie J. Allen ${ }^{2}$, and David W. McComb ${ }^{1}$ \\ 1. Department of Materials Science and Engineering, The Ohio State University, 477 Watts Hall, 2041 \\ College Road, Columbus, OH 43210, USA \\ 2. School of Physics, University of Melbourne, Parkville, Victoria 3010, Australia
}

Advances in aberration corrected scanning transmission electron microscopy (STEM) have allowed for sub-Ångstrom probes and atomic resolution imaging. High-angle annular dark-field (HAADF) STEM produces images with contrast related to the average atomic number $(Z)$ of the species in a given column, which can be useful in characterizing local ordering phenomena. However, to fully exploit the characterization potential of HAADF-STEM experimental images must be examined in conjunction with simulated images. Comparisons between experiment and simulation facilitates the identification of the underlying structure of the specimen in the presence of the confounding effects of dynamical scattering, thermal diffuse scattering (TDS), and other inelastic processes [1]. Experimentally, quantitative HAADF-STEM imaging is non-trivial but offers the ability to capture spatially resolved chemical information with atomic resolution [2].

In the developing field of spintronics, it has been difficult to find materials systems with properties that lend themselves to the use of electron spin for read/write capabilities in solid state devices [3]. Half-metallic double perovskites, of the form $\mathrm{A}_{2} \mathrm{BB}^{\prime} \mathrm{O}_{6}$, have been found to be of particular interest in the field of spintronics because they exhibit high spin polarization and Curie temperatures well above room temperature $[4,5]$. High-quality, double perovskite epitaxial thin films are essential to realizing the full potential of spin injectors, which in turn is highly reliant on the degree of $\mathrm{B} / \mathrm{B}^{\prime}$ site ordering within the film. Here we shall consider two different types of materials in the double perovskite family: $\mathrm{Sr}_{2} \mathrm{FeMoO}_{6}(\mathrm{SFMO})$ and $\mathrm{Sr}_{2} \mathrm{CrReO}_{6}(\mathrm{SCRO})$.

In the double perovskite crystal structure, SCRO, single species columns of $\mathrm{Cr}$ and $\mathrm{Re}$, as well as mixed species columns of $\mathrm{Sr} / \mathrm{O}$, form along the $<100>$ direction. In a linear imaging model, the intensity is based on the average $\mathrm{Z}$ within the column, this structure, when perfectly ordered, provides three distinct intensity levels with Re columns having the highest intensities $\left(Z_{\mathrm{Re}}=75\right), \mathrm{Sr} / \mathrm{O}$ columns intermediate intensities $\left(Z_{\mathrm{Sr} / \mathrm{O}}=27\right)$ and $\mathrm{Cr}$ columns the least intense $\left(\mathrm{Z}_{\mathrm{Cr}}=24\right)$, Fig. 1. However, experimentally it has been seen that some regions of the SCRO thin film, typically near an interface with the substrate, do not exhibit three distinct columnar intensities, but instead have atomic columns of similar intensity, Fig. 2. This observation would seem to point towards varying degrees of atomic ordering on the local level.

In this work we used the quantum excitation of phonons model [6] to simulate HAADF-STEM images of double perovskite thin film samples with varying degrees of B/B' disorder. By varying the amount of local disorder introduced into the system, the affect that disorder has on the intensity of the atomic columns was studied. These types of calculations can be used in comparison with experimental images where local disorder is observed, the result of which will allow for a better understanding of the relationship between the processing, crystal structure, and physical properties of these thin films. Results 
and preliminary comparisons to experimental images will be presented and discussed for a quantitative determination of $\mathrm{B} / \mathrm{B}^{\prime}$ disorder in SFMO.

\section{References:}

[1] SD Findlay, DO Klenov, S Stemmer, LJ Allen, Mat. Sci. and Tech. 24 (2008) p.660

[2] JM LeBeau and S Stemmer, Ultramicroscopy 108 (2008) p.1653

[3] AJ Hauser, et al, Phys. Rev. B 85 (2012) p.161201(R)

[4] KL Kobayashi, et al, Nature 395 (1998) p.677.

[5] JM Serrate, De Teresa, and MR Ibarra, J. Phys: Condens. Matter 19 (2007) p.023201.

[6] BD Forbes, etal, Phys. Rev. B. 82 (2010) p. 104103.

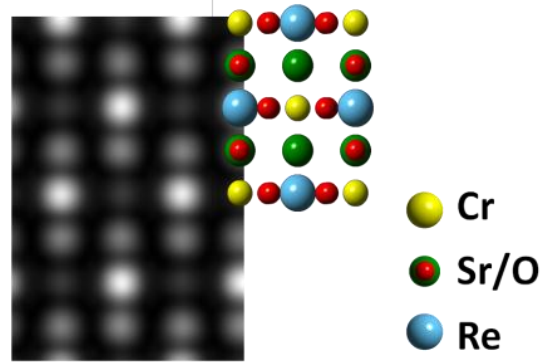

Figure 1: Simulated HAADF-STEM image and crystal structure of perfectly ordered $<100>$ SCRO

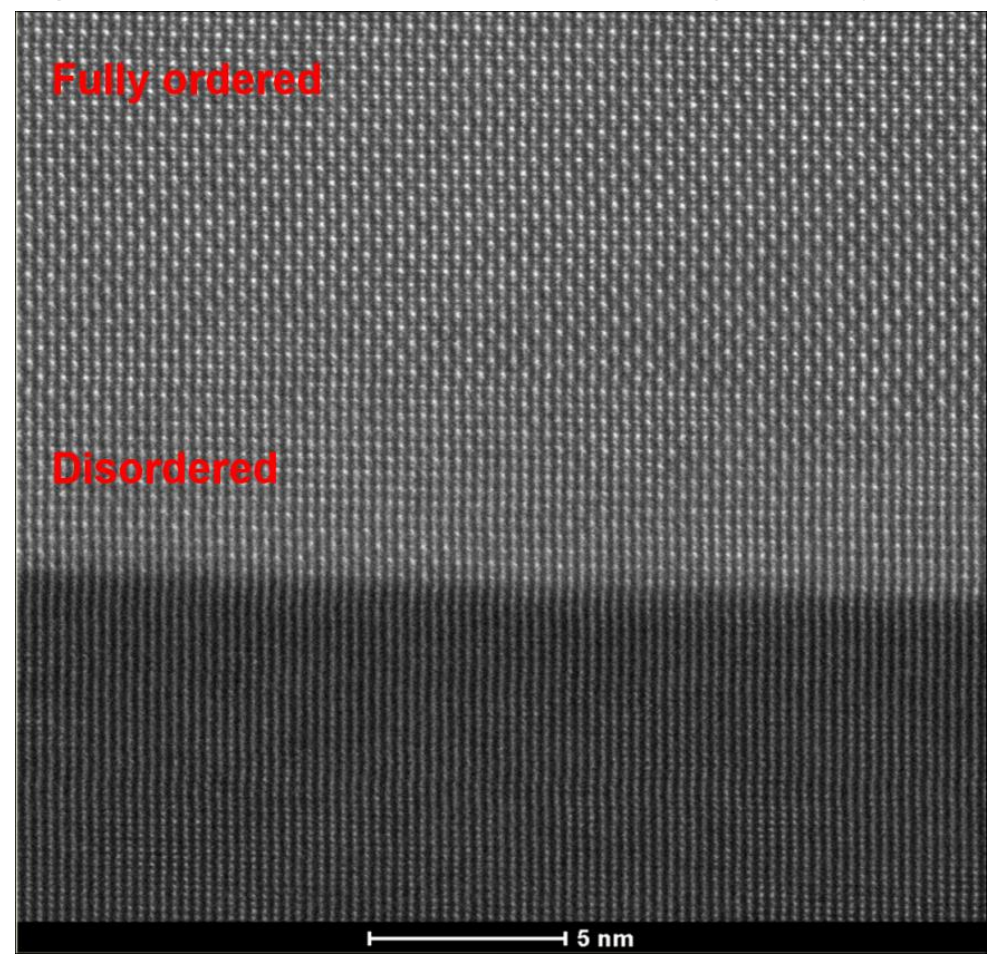

Figure 2: Experimental HAADF-STEM image of $<100>$ SCRO (top layer) exhibiting apparent local disorder at the interface transitioning to fully ordered further from the interface. 